\title{
Turbulence and Laminar Structures: Can they co-exist?
}

\author{
V.M. Canuto ${ }^{1,2}$ \\ ${ }^{1}$ NASA, Goddard Institute for Sirace Studies, \\ 2880 Broadway, New York, N.Y., 10025 \\ ${ }^{2}$ Dept. of Applied Physics and Mathematics, \\ Columbia University, New York, N.Y., 10027
}

\begin{abstract}
Schwarzschild first suggested that the laminar structures observed in the high Reynolds number $\operatorname{Re}=\mathrm{UL} / \nu \approx \mathrm{O}\left(10^{12}\right)$ solar photosphere are the result of turbulence rather than a proof of its absence. He reasoned that since turbulence generates large turbulent viscosities $\nu_{\mathrm{t}}>>\nu$, the "effective" Reynolds number $\mathrm{Re}=\mathrm{UL} / \nu_{\mathrm{t}} \approx \mathrm{O}(1)$. Schwarzschild's argument is, however, incomplete for it assumes that the entire role of the non-linear interactions is to "enhance" viscosity. While this is not true in general, we present a proof of how and why it may occur, thus completing Schwarzschild's argument. We further discuss the fact that the same model non-local turbulence models have been shown to reproduce LES data for a variety of flows pertaining to astrophysics, geophysics and laboratory situations (at a fraction of the time).
\end{abstract}




\section{The problem.}

Recently, questions have been raised as to the compatibility of the generally accepted notion of turbulence with the existence of solar atmospheric structures that exhibit laminar features. It is important to notice at the outset that such structures are not a recent theoretical discovery or prediction since the knowledge of their existence goes back to 1890 (Minnaert 1958). To avoid unproductive epistemological discussions, we suggest to replace the term turbulence with the more general term non-linear interstions (NLI), of which turbulence is a manifestation. The question can be rephrased as: are laminar structures compatible with the NLI? The inconsistency that has beset recent discussions is the assumption that the relation

$$
\mathrm{NLI}=0 \rightarrow \text { Laminar Structures }
$$

also implies the converse:

$$
\text { Laminar Structures } \rightarrow \mathrm{NLI}=0
$$

We shall show that (1a) is correct but (1b) is not: laminar structures are compatible with NLI or with the presence of turbulence understood in the most general terms.

Schwarzschild (1959) was the first to propose an explanation for the observed laminar features in the Re>>1 solar atmosphere. He reasoned that the laminar structures are due to turbulence since the latter enhances the molecular viscosity $\nu$ to

$$
\nu \mapsto \nu+\nu_{\mathrm{t}} \sim \nu_{\mathrm{t}} \sim \epsilon^{1 / 3} \ell^{4 / 3}
$$

where we have taken $\nu_{\mathrm{t}}>>\nu$ and used the well known Richardson's law for $\nu_{\mathrm{t}}(\epsilon$ is the rate of energy input into the system). Eq.(2a) tells us that, contrary to the molecular viscoity which is size independent, the effective viscosity felt by a structure of size $\ell$ depends on $\ell$. Thus, the larger the structure, the larger the viscosity it feels. Recalling one of the most well established experimental facts in Re $>>1$ flows (Tennekes and Lumley, 1972)

$$
\epsilon \sim U^{3} \ell^{-1}
$$

we obtain $\nu_{\mathrm{t}} \sim \mathrm{U} \ell$ which, once substituted into the Reynolds number, yields: 


$$
\mathrm{Re}=\frac{\mathrm{U} \ell}{\nu} \rightarrow \frac{\mathrm{U} \ell}{\nu+\nu_{\mathrm{t}}} \sim \mathrm{O}(1)
$$

Any proposed model for the laminar structures that is different from Schwarzschild's must first prove that the latter is untenable. However, Schwarzschild's model is not cited and thus not disproved, in a recent work (Nordlund et al., 1997) where turbulence concepts are deemed irrelevant to explain the observed laminar structures.

Recently, Stothers (2000) has revisited the problem, has extended Schwarzschild's argument also to the deep layer of the convective zone and has suggested that in fact the existence of laminar-like structures may well be due to the effect of turbulence rather than to the absence of it.

\section{i. The full argument.}

Though physically attractive, the Schwarzschild-Stothers argument is incomplete for it rests on the unproven assumption that the only role of the NLI is to renormalize the nolecular viscosity to a dynamical value $\nu_{\mathrm{d}}(\mathrm{k})$ which, rather than being a constant like the molecular $\nu$, depends on the size of the structure one considers $\left(\mathrm{k} \sim \ell^{-1}\right)$ :

$$
\nu \rightarrow \nu_{\mathrm{d}}(\mathrm{k}) \equiv \nu+\nu_{\mathrm{t}}(\mathrm{k})
$$

Eq.(4), with a specific form for $\nu_{\mathrm{t}}(\mathrm{k})$, was first suggested by Heisenberg and Obukhov and shown to reproduce the Kolmogorov spectrum. In the present context such a spectrum is irrelevant since we deal with structures much larger than those of the so-called inertial regime and thus, the existence or not of a Kolmogorov spectrum is inconsequential (see, however, Fig.1 of Chan and Sofia, 1996). The renormalization (4) is only part of the whole effect of the NLI. It represents the effect on a given eddy $\mathrm{k}$ of all the smaller eddies and is thus an UV (ultraviolet) effect. We are not interested in them since we focus on the other part of the spectrum, the IR (infrared) part where the large scale structures are found. The key question then becomes: How are these large scales affected by the NLI? This is the part of the problem missing in Schwarzschild's original argument. 


\section{An exact solution of the Navier-Stokes Equations.}

Let us call $u_{j}(\mathbf{k})$ the general stochastic (random) solution of the Navier-Stokes equations (NSE) and let us consider the physically measurable correlation function,

$$
\mathrm{Q}_{\mathrm{ij}}\left(\kappa, \kappa^{\prime}\right)=\left\langle\left(\mathrm{u}_{\mathrm{i}}(\kappa) \mathrm{u}_{\mathrm{j}}\left(\kappa^{\prime}\right)\right\rangle\right.
$$

where $\kappa=\mathbf{k}, \omega$. In 1961, Wyld derived a formally exact solution of the NSE under the assumption of homogeneity. His result for the trace of $\mathrm{Q}_{\mathrm{ij}}$, valid for an arbitrary Reynolds number, is as follows:

$$
\mathrm{Q}(\kappa)=\frac{\phi^{\mathrm{ext}}(\mathrm{k})+\phi^{\mathrm{turb}}(\mathrm{k})}{\omega^{2}+\mathrm{k}^{4} \nu_{\mathrm{d}}^{2}(\mathrm{k})}
$$

This result $i$. often cited as the Wyld-Dyson equation because is exact in the same sense as the Dyson e fuations for QED: they are formally exact in that they exhibit what NLI do but do not provide the tools to compute the ingredients. In the QED case, the new ingredients we the polarization (or dielectric constant) and mass tensors. Here, one observes the interesting fact that the effect of the NLI is twofold: 1) they renormalize the molecular viscosity exactly as in Eq.(4) but, more importantly for our discussion, 2) the large scales are also renormalized in the sense that the correlation of the arbitrary external forces

$$
\phi_{i j}^{e x t} \equiv\left\langle\mathrm{f}_{i}^{e x t} \mathrm{f}_{j}^{\mathrm{ext}}\right\rangle
$$

is changed to:

$$
\phi_{i j}^{e x t} \rightarrow \phi_{i j}^{e x t}+\phi_{i j}^{t}
$$

Thus, the "true force" felt by an eddy is not only the external force (e.g., buoyancy, shear) but also an additional $\mathrm{f}_{\mathrm{i}}^{\mathrm{t}}$ whose correlation function is $\phi_{\mathrm{j}}^{\mathrm{t}}$. This renormalization is the more interesting of the two since is telling us that the NLI give rise to a "ghost force" $f_{j}^{t}$ which operates even when the external forces are no longer relevant, for example in the inertial

range. Without $\phi_{\mathrm{ij}}^{\mathrm{t}}$, one could not explain why eddies that are no longer affected by the external forces do not exhibit an energy equipartition spectrum but a spectrum of the form $\mathrm{E}(\mathrm{k}) \sim \mathrm{k}^{-\mathrm{n}}(\mathrm{n}=5 / 3,3, \mathrm{etc})$. 
It is important to realize that (6a) is also the solution of the following stochastic, Langevin-type equation for the random velocity $\mathrm{u}_{\mathrm{i}}(\mathbf{k})$

$$
\frac{\partial}{\partial t} \mathrm{u}_{\mathrm{i}}(\mathbf{k})=\mathrm{f}_{\mathrm{i}}^{\mathrm{t}}(\mathbf{k})+\mathrm{f}_{\mathrm{i}}^{e x t}(\mathbf{k})-\mathrm{k}^{2} \nu_{\mathrm{d}}(\mathrm{k}) \mathrm{u}_{\mathrm{i}}(\mathbf{k})
$$

provided the external and turbulent forces do not correlate. Eq.(7a) substitutes for the original NSE and exhibits an important feature: while the original NSE are non-linear in the velocity fields and linear in the coefficients (which are actually constants), Eq.(7a) is linear in the velocity field but non-linear in the coefficients. In practical calculations of real turbulent flows, one does not need the form of the turbulent forcing $f_{i}^{t}$ but only the work performed by it, which is obviously defined as:

$$
A^{t}(k)=k^{2} \int d \Omega_{k} d k^{\prime}<u_{i}\left(k^{\prime}\right) f_{i}^{t}(k)>
$$

The equation for the Reyı.olds stresses $R_{i j}$

$$
\mathrm{R}_{\mathrm{ij}}(\mathbf{k}, \mathrm{t})=\left\langle\mathrm{u}_{\mathrm{i}}(\mathbf{k}) \mathrm{u}_{\mathrm{j}}(\mathbf{k})>\right.
$$

can easily be derived from (7a) to be:

$$
\frac{1}{2} \frac{\partial}{\partial t^{2}} R_{i j}(\mathbf{k})=A_{i j}^{t}(k)-k^{2} \nu_{d}(k) R_{i j}(k)+A_{i j}^{e x t}(\mathbf{k})
$$

In Canuto and Dubovikov (1996a,b,c) it was shown that $\left(\mathrm{P}_{\mathrm{ij}}\right.$ is the projection operator):

$$
\begin{aligned}
& A_{i j}^{t}(k)=\left(8 \pi k^{2}\right)^{-1} P_{i j}(k) A^{t}(k) \\
& A^{t}(k)=-\Pi(k) E^{-1}(k) \frac{\partial}{\partial k} E(k)
\end{aligned}
$$

Eq.(7f) agrees with the general result derived by Monin and Yaglom (1971):

$$
\phi^{\text {turb }}(\mathrm{k}) \sim \mathrm{k}^{-2} \mathrm{E}^{-1}(\mathrm{k}) \frac{\partial}{\partial \mathrm{k}} \mathrm{E}(\mathrm{k})
$$

For a model of turbulence to be viable, it must be able to reproduce the large variety of data now available for flows of different nature. Model ( $7 \mathrm{~d}-\mathrm{f})$ has been tested over more than 80 turbulent statistics characterizing: freely decaying flows, shear generated flows, buoyancy generated flows, 2D flows, turbulent flows under rotation, buoyant flows under rotation, etc (Canuto et al., 1996, 1997, 1999). For example, in $\operatorname{Re}>>1$ shear flows, the Reynolds stress spectrum predicted by the above model:

$$
\mathrm{R}_{12}(\mathrm{k})=-\mathrm{A \epsilon}^{1 / 3} \mathrm{Sk}^{7 / 3}
$$

matches very closely the measured data of Saddoughi and Veravalli (1994). The same 
model was used in an astrophysical setting to explain the observed Reynolds stresses at the surface of the sun (Canuto et al., 1994a) while the comparison of the model results with LES data, including the topology of up/down drafts of the convective regime, has been successfully carried out by Kupka (1999).

\section{A special group of eddies.}

Inspection of (7f) shows that given an energy spectrum $\mathrm{E}(\mathrm{k})$ of arbitrary shape there must exist a subset of eddies near the maximum of the energy spectrum for which

$$
\frac{\partial}{\partial \mathrm{k}} \mathrm{E}(\mathrm{k}) \sim 0
$$

in which case the ghost forcing vanishis

$$
\phi_{i j}^{\text {turb }} \rightarrow 0
$$

and the Reynolds stresses satisfy an equation which is analogous to that of the linear case with an enhanced viscosity,

$$
\frac{1}{2} \frac{\partial}{\partial t} R_{i j}(\mathbf{k})=A_{i j}^{e x t}(\mathbf{k})-k^{2} \nu_{d}(k) R_{i j}(\mathbf{k})
$$

For this group of eddies, and only for them, the whole effect of the NLI reduces to the renormalization of the viscosity (4). Thus, the following can be stated:

In an arbitrary Re $>>1$ flow, the NLI affect both small and large scales. For the latter, there is a subset of eddies that feel only the effect of an enhanced viscosity and thus, they behave as if they were governed by the standard laminar flow equations but with an enhanced $\nu$ which makes their effective Re 1. This completes Schwarzschild's argument.

\section{A further test}

Our argument is however not yet complete. We must still show that our model can not only justify Schwarzschild-Stothers argument but is also capable of reproducing DNS/LES data. Otherwise it could be (somewhat perversely) argued that the above result is the outcome of a peculiar model. There is also another reason. In studies of stellar convection, the impression has been created that LES have revealed a set of completely 
new phenomena that no other formalism is capable of reproducing. It has even been suggested that there is a new paradigm (Spruit, 1999). We fail to understand this conclusion primarily because the same physical phenomena described by stellar LES are known to occur in geophysics where no new paradigm was invoked. First, as Lumley has pointed out (1990), non-local, second-order closure models (SOC models for short) are capable of reproducing the major features (global and topological) of turbulent flows. If, however, one arbitrarily assumes SOC to mean only second-order moments (the local limit), then important effects are arbitrarily left out and the model becomes incapable of reproducing any topological feature. But this is the outcome of an arbitrary definition of the SOC model not required by the model itself. Comparing LES results with a local model is a biased procedure leading to a foregone conclusion for it employs a turbulence model that does not contain what is required for a fair comparison. As Lumley (1990) has remarked, it is like a Fourier representation that cmits all Fourier modes above the second and leaves out much of interest in the function. Lumley (1981) has shown that use of third and fourth order moments is indeed the key to capture information about phases. To further prove the point, we cite several examples.

1) Petrovay (1990) showed that even the local MLT plus use of third-order moments can explain the major topological features of convective motions,

2) non-local SOC models reproduce the major results of three different LES for the convective planetary boundary layer, specifically, updrafts, donwdrafts, filling factors, etc. (Canuto et al., 1994b; Canuto and Cheng, 2000)

3) within the astrophysical context, Kupka (1999) has shown that the LES convective fluxes and down/up drafts are also reproduced by non-local SOC models,

4) a list of some 80 laboratory and/or numerically computed turbulent statistics belonging to a variety of turbulent flows has been reproduced by such models (Canuto and Dubovikov, 1996-1999),

5) in the case of stellar convection, the flux conservation law reads 


$$
F(\text { conv })+F(\operatorname{rad})+F(\kappa e)=\text { constant }
$$

where $F(\kappa e)$ is the flux of turbulent kinetic energy. Since we can write that

$$
\mathrm{F}(\text { conv })=\mathrm{F}^{\mathrm{u}}(\text { conv })+\mathrm{F}^{\mathrm{d}} \text { (conv) }
$$

where the $\mathrm{u} / \mathrm{d}$ stand for up and down fluxes, and since (Chan and Sofia, 1986, 1989; Cattaneo et al., 1991) numerical results show that

$$
\mathrm{F}^{\mathrm{d}}(\text { conv })+\mathrm{F}(\kappa e) \approx 0
$$

we are left with

$$
\mathrm{F}^{\mathrm{u}}(\text { conv })+\mathrm{F}(\mathrm{rad})=\text { constant }
$$

where $\mathrm{F}^{\mathrm{u}}$ (conv) represents the broad updrafts of disordered notion that the SOC models traditionally compute. Thus, LES lead not to the conclusior that turbulence models have become irrelevant, but to the contrary, that they offer the way io compute the only left-over mechanism to transfer heat. A key ingredient is of course the large cancellation (10c) with the consequence that the only transport left is by the broac. updraft motion. This same point was first made by Cattaneo et al. (1991) in their conclusions.

There is, however, a larger issue, regarding the origin and universality of the down/up drafts picture. In this context, it is important to recall that these topologies are well known in geophysics, specifically in the study of the strongly convective planetary boundary layer (Deardorff, 1974, 1980, 1985; Willis and Deardorff 1976; Wyngaard and Brost, 1984; Moeng, 1984; Wyngaard, 1987; Schmidt and Schumann, 1989; Moeng and Wyngaard, 1989). As for universality, we recall that one must distinguish three cases (Moeng and Rotunno, 1990):

1) convection due to heating from below: this corresponds to the layer above the earth's surface (without clouds). SOC models and LES show that the skewness of the velocity field:

$$
S_{w}=\overline{w^{3}}\left(\bar{w}^{2}\right)^{-3 / 2}
$$

is positive

$$
\mathrm{S}_{\mathrm{w}}>0
$$


and since the filling factors $\sigma$ of the updrafts (1- $\sigma$ for the downdrafts) given by

$$
\sigma=\frac{1}{2}\left[1-\mathrm{S}_{\mathrm{W}}\left(4+\mathrm{S}_{\mathrm{W}}^{2}\right)^{-\frac{1}{2}}\right]
$$

implies that:

$$
\sigma<1 / 2
$$

which corresponds to narrow updrafts and broad downdrafts. These predictions are in full agreemeni with the measured data (LeMone, 1989; Zilitinchevich et al., 1999, fig.1).

2) ccivection due to cooling from above: this corresponds to the case of the ocean or to the atmosphere topped with clouds which radiatively cool it (Nicholls and Leighton, 1986; Moeng and Schumann, 1991). In this case, the skewness $S_{w}$ is negative anc one has narrow downdrafts and broad updrafts. Clearly, this physical situation is the one that corresponds more closely to what happens in the upper part of the solar convective zor e where there is cooling by radiative losses,

3) convection due to heating from below and cooling from above: this is tise most natural state of the atmosphere with cooling clouds as well as the situation in stars where there is heating from below and cooling from above due to radiative losses. Such a situation is also found in Rayleigh-Benard convection. The resulting topology is understood as i combination of the skewness distribution of the bottom-heating and top-cooling cases.

In conclusion, non-local SOC models have all the physical ingredients and have been explicitly shown, to reproduce LES data with the added conspicuous advantage of being extremely flexible and of requiring a minute fraction of the computer time required by any LES. From the physical viewpoint, these studies have shown that convection exhibits the same general features irrespectively of whether it occurs in a star or in the earth's atmosphere, a "unification" that we deem much more scientifically valuable than any priority claim, even if the latter were true.

\section{Conclusions}

We have shown that (1a) is correct while (1b) is not. In fact, in any $R e>>1$ flow 
where the NLI are important, there exists a group of large scale structures for which the NLI effect is only to enhance viscosity. These scales are governed by a linear dynamics not because there are no NLI but because there are. This completes Schwarzschild-Stothers argument which assumed, without proof, that NLI only produce (4). Laminar structures can co-exist with smaller eddies that behave in a way described by turbulence models.

We find no reason to suggest that astrophysical LES have given us a new vision of convection to call for a new paradigm. In fact, the same physical situation is found in other contexts. Specifically, convection in the earth's surface boundary layer, with heating from below, radiative cooling from above and a strong density decrease, has been known to jive rise to topological structures which have also been found in the stellar case because of the similarity of the basic physical mechanisms that operate. It is therefore not a questio. . of priority but a confirmation of general features of convective flows. As to the non-local SOC models, they have matched LES data quite well, they require a fraction of the t.me required by the LES and are the only ones with a realistic chance of being hooked-up io a stellar structure/atmosphere code.

\section{Acknowledgements}

The author would like to express his thanks Dr.R.B.Stothers for many useful discussions on the topic of this paper.

\section{References}

Canuto, V.M., F.O. Minotti and O. Schilling, 1994a, ApJ, 425, 303,

Canuto, V.M., F.O. Minotti, R. Ronchi, R.M. Ypma, and O. Zeman, 1994b, J.Atm. Sci. 51, 1605

Canuto, V.M. and M.S. Dubovikov, 1996a, Phys. Fluids, 8, 571; 1996b, ibd., 1996b, 8, 587; 1996c, ibd., 8, 599

Canuto, V.M., M.S. Dubovikov and A. Dienstfrey, 1997a, Phys. Fluids, 9, 2118; 1997b, ibd. 
1997b, 9, 2132; 1997c, ibd., 9, 2141

Canuto, V.M. and M.S. Dubovikov, 1999a, Phys. Fluids, 11, 659; 1999b, ibd., 1999b, 11, $665 ; 1999 \mathrm{c}$, ibd., 11,678

Canuto, V.M. and Y. Cheng, 2000, J. Atm. Sci. (submitted)

Cattaneo, F., N.E. Brummel, J. Toomre, A. Malagoli and N.E. Hurlburt, 1991, ApJ, 370, 282

Chan, K.L. and S. Sofia, 1996, ApJ, 466, 372

Deardorff, J.W., 1974, Boundary Layer Meteor., 7, 199; ibid., 1980, 18, 495; ibid., $1985,32,205$

Kupka, F, 1999, ApJ Letters, 526, L45

LeMone, M.A., 1989, J. Atm. Sci., 47, 1163

Lumley, J.L., 1981, in Transition and Turbulence, ed. R. Meyer, New York, Academic

Lumley, J.L., 1990, in Whither Turbulence, J.L. Lumley Editor, Springer-Verlag, New York, pg. 49

Minnaert, M. 1958, in The Sun, Ed. by G.P. Kuiper, Univ. Chicago Press, Chicago, Ill.

Moeng, C.H., 1984, J. Atm. Sci., 41, 2052

Moeng, C.H. and J.C. Wyngaard, 1989, J. Atm. Sci., 46, 2311

Moeng, C.H. and R. Rotunno, 1990, J. Atm. Sci., 47, 1149

Moeng, C.H and U. Schumann, 1991, J. Atm. Sci., 48, 2280

Monin A.S and A.M. Yaglom, 1971, Statistical Fluid Mechanics, MIT Press, Cambridge, Mass.

Nicholls, S. and J. Leighton, QJRMS, 112, 4311986

Nordlund, A., H.C. Spruit, H.G. Ludwig, and R. Trampedach, 1997, A\&A, 328, 229

Petrovay, K.G., 1990, ApJ, 362, 722

Saddoughi, S.G. and S.V. Veravalli, 1994, JFM, 268, 333

Schmidt, H. and U. Schumann, 1989, JFM, 200, 511

Schwarzschild, M., 1959, ApJ, 130, 345 
Spruit, H.C., 1999, Italian Astron. Society Proceed. (in press)

Stothers, R.B., 2000, MNRAS (in press)

Tennekes H. and J.L. Lumley, 1972, A First Course in Turbulence, MIT Press, Cambridge, Mass.

Wyld, H.W., 1961, Ann. Phys., 14, 143

Willis, G.E., and J.W. Deardorff, 1976, QJRMS, 102, 427

Wyngaard, J.C. and R.A. Brost, 1984, J. 4tm. Sci., 41, 102

Wyngaard, J.C., 1987, J. Atm. Sci., 44, 1083

Zilitinkevich, S.S., D.V. Mironov, V.M. Gryanik, V.N. Lykossov, and 1999, J. Atmos.

Sci., 56, 3463 